\title{
TESTFIELD TRENTO: GEOMETRIC EVALUATION OF VERY HIGH RESOLUTION SATELLITE IMAGERY
}

\author{
G. Agugiaro ${ }^{\text {a }}$, D. Poli ${ }^{\text {b }}$, F. Remondino ${ }^{\mathrm{a}}$ \\ a 3D Optical Metrology unit, Bruno Kessler Foundation, Trento, Italy \\ \{agugiaro, remondino\}@fbk.eu, http://3dom.fbk.eu \\ ${ }^{\mathrm{b}}$ Vermessung AVT ZT-GmbH, Austria \\ d.poli@terra-messflug.at, http://www.avt.at
}

Commission I - WG4

KEY WORDS: Testfield, Very High Resolution, GeoEye-1, WorldView-2, Digital Surface Modelling, Quality Analysis

\begin{abstract}
:
Today the use of spaceborne Very High Spatial Resolution (VHSR) optical sensors for automatic 3D information extraction is increasing in the scientific and civil communities. The 3D Optical Metrology (3DOM) Unit of the Bruno Kessler Foundation (FBK) in Trento (Italy) has collected stereo VHSR satellite imagery, as well as aerial and terrestrial data over Trento, with the aim to create a complete data collection with state-of-the-art datasets for investigations on image analysis, automatic digital surface model (DSM) generation, 2D/3D feature extraction, city modelling and data fusion. The testfield region covers the city of Trento, characterised by very dense urban (historical centre), residential and industrial areas, and the surrounding hills and steep mountains (approximate height range 200-2100 m) with cultivations, forests and bare soil.

This paper reports the analysis conducted in FBK on the VHSR spaceborne imagery of Trento testfield for 3D information extraction. The data include two stereo-pairs acquired by WorldView-2 in August 2010 and by GeoEye-1 in September 2011 in panchromatic and multispectral mode, together with their original Rational Polynomial Coefficients (RPC), and the position and description of well distributed ground points. For reference and validation, a DSM from airborne LiDAR acquisition is used. The paper gives details on the project and the dataset characteristics. The results achieved by 3DOM on DSM extraction from WorldView-2 and GeoEye-1 stereo-pairs are shown and commented.
\end{abstract}

\section{INTRODUCTION}

The 3D Optical Metrology (3DOM) unit of the Bruno Kessler Foundation (FBK) in Trento (Italy) is building a testfield by collecting high resolution (HR) and very high resolution (VHR) satellite imagery, airborne and UAV images and orthophotos, LiDAR scans, and a network of circa 70 ground control points. The testfield region is centred on the city of Trento, characterised by an historical centre, residential and industrial areas and is surrounded by hills and steep mountains, with a variety of differently agricultural areas, forests and bare soil.

The aim is to build state-of-the-art datasets to test methods and algorithms for image radiometric analysis, triangulation and geo-referencing, automatic surface modelling, 2D/3D feature extraction, change detection and data fusion.

This paper focuses on the processing of the very high resolution (VHR) satellite imagery available in the testfield for digital surface model (DSM) extraction. Spaceborne VHR optical sensors are able to scan any point of the Earth surface with a ground spatial resolution around half meter and a revisit time in the order of few days; through their very agile manoeuvring they can also acquire stereo data within the same orbit just using the CCD line combination, by pointing at the same area from two or more orbit positions (Poli and Toutin, 2012). Thanks to the improved acquisition technology, this new class of VHR sensors allows surface modelling up to building level of detail, in 2.5D (DSM) or even in 3D (object extraction) (Poli et al. 2009; Arefi and Reinartz, 2011; d'Angelo and Reinartz, 2011; Capalbo et al., 2012; Poli and Caravaggi, 2012).

The paper introduces the project, with information on the testfield characteristics and activity plan, and describes the processing carried out on WorldView-2 and GeoEye-1 stereopairs. The images were oriented using the available ground control points and digital surface models were generated with area- and feature-based least-square image matching. The DSMs were compared both qualitatively (visual analysis) and quantitatively, using the LIDAR as reference. The results are presented and commented.

\section{TEST AREA AND DATA SOURCES}

The test area is located in Trento, a city of about 115.000 inhabitants in the Trentino-Alto Adige region (Northeast of Italy). It lies on the banks of the river Adige in the homonymous valley (North-South direction) and is surrounded East and West, by the Alps (Fig. 1). The testfield varies from urban areas with residential, industrial and commercial buildings at different sizes and heights, to agricultural or forested areas, and rocky steep surfaces, offering therefore a heterogeneous landscape in term of geometrical complexity, land use and cover. The city of Trento lies circa $200 \mathrm{~m}$ a.s.l., while the nearby peaks reach as high as $2100 \mathrm{~m}$.

\subsection{Data sources}

Several heterogeneous datasets were identified or collected at varying spatial resolution, ranging from satellite imagery to aerial imagery, LiDAR data, GNSS surveyed points, GIS data, etc. A global overview about the extents of the areas covered by each type of imagery data is given in Fig. 1. For completeness, existing data available also from other institutions on the territory (e.g. the Autonomous Province of Trento, the Municipality of Trento, RSLab of the University of Trento) are mentioned in the following list.

The spaceborne data are summarised in Table 1 and include:

- WorldView-2 (WV2) stereo-pair: the processing level is Stereo $1 \mathrm{~B}$, i.e. the images are radiometrically and sensor corrected, but not projected to a plane using a map projection or datum, thus keeping the original acquisition geometry. The available channels are the panchromatic one and eight multispectral ones. In addition to the standard multispectral bands (blue, green, red, near infrared), 


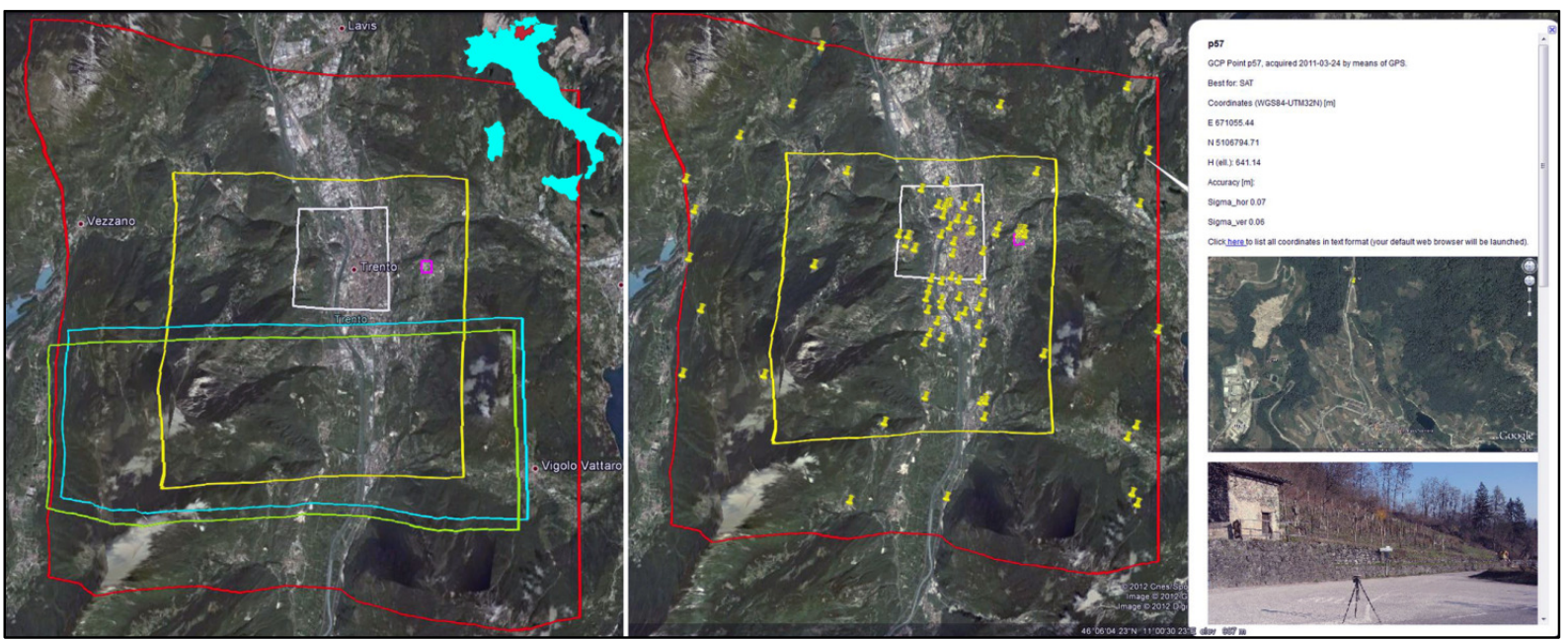

Figure 1. [Left] Approximate spatial extents of satellite and aerial imagery over the Trento testfield. The extents of each imagery type projected onto the DSM: WorldView-2 stereo-pair (red), GeoEye-1 stereo-pair (yellow), Quickbird scenes (green and cyan), aerial (white) and UAV (violet). SPOT imagery is not depicted, as it comprises the whole area. [Right] Distribution of ground points with respect to WorldView-2 (red) and GeoEye-1 (yellow) imagery extent, with corresponding metadata and photographic information.

WorldView-2 scans in the coastal (400-450 nm), yellow $(585-625 \mathrm{~nm})$, red edge $(705-745 \mathrm{~nm})$ and near infrared-2 $(860-1040 \mathrm{~nm})$ spectral ranges. The images cover an area of $17.64 \times 17.64 \mathrm{~km}$ and have an overlap of $100 \%$. The images were provided with Rational Polynomial Coefficients (RPCs).

GeoEye-1 (GE1) stereo-pair: the stereo images have 100\% overlap and are provided as GeoStereo product, that is, they are projected to a constant base elevation. The available bands are the panchromatic one and four multispectral bands (blue, green, red, and near infrared). The images cover an area of $10 \times 10 \mathrm{~km}$. For each image the RPCs were provided.

- Quickbird images: two single scenes were acquired on 30 October 2005 and 17 July 2006, respectively. The panchromatic and multispectral channels are available.

SPOT-5 images: a scene was acquired over Trento on 31 August 2008 with the HRG sensor (2.5 m GSD, panchromatic) and VEGETATION sensor (10 m GSD, multispectral).

The airborne data include:

- A raster-based DSM (with the filtered DTM) derived from a LiDAR flight in $2006 / 7$ at ca. 1.3 points $/ \mathrm{m}^{2}$. The DSM has a grid space of $1 \mathrm{~m}(2 \mathrm{~m}$ in some scarcely inhabited mountainous areas). The height accuracy for the original LiDAR data is given as $\sigma_{z}=15 \mathrm{~cm}$ for the DSM, and $\sigma_{z}=30$ $\mathrm{cm}$ for the DTM. The LiDAR DSM was considered the reference dataset for the geometric analyses.

- A set of 230 digital orthophotos produced in 2006 and 2009, with a ground sample distance $(\mathrm{GSD})$ of $50 \mathrm{~cm}$.

- A block of 10 nadir aerial images acquired in 2009 with a RMKTOP15 analogic camera over the city of Trento (overlap of $60 \%$ along track and $30 \%$ across track). The digitised images have an average GSD of ca. $12 \mathrm{~cm}$.

A block of about 180 UAV images acquired in January 2012 with a Microdrones MD4-200 mounting a Pentax Optio A40 digital camera (overlap of $80 \%$ along track and $40 \%$ across track). The images have an average GSD of ca. $3 \mathrm{~cm}$.

Other data include:

- About 70 ground points, acquired in spring/summer 2011 by means of a GNSS receiver and post-processed using the
Trento GNSS permanent station in order to achieve subdecimetre accuracy.

- 12 ground points, located on building corners and roofs acquired in January 2012 by Total Station.

- GIS vector data for the whole province of Trento, consisting (mainly) of a topographic map at nominal scale of 1:10000, and a set of 447 cadastral maps in vector format at nominal scale of 1:1000.

Table 1. Characteristics of satellite imagery in the Trento testfield. PAN: panchromatic, MS4: multispectral (4 bands), MS8: multispectral (8 bands), GSD: Ground Sample Distance.

\begin{tabular}{|c|c|c|c|c|c|c|}
\hline Sensor & $\begin{array}{c}\text { Acquisition } \\
\text { date }\end{array}$ & Bands & $\begin{array}{c}\text { Avg. } \\
\text { GSD } \\
{[\mathbf{m}]}\end{array}$ & $\begin{array}{c}\text { Size x } \\
{[\mathbf{k m}]}\end{array}$ & $\begin{array}{c}\text { Size y } \\
{[\mathbf{k m}]}\end{array}$ & Licensee \\
\hline Quickbird & 30 Oct 2005 & $\begin{array}{c}\text { PAN } \\
\text { MS4 }\end{array}$ & $\begin{array}{c}0.6 \\
2.4\end{array}$ & 14,75 & 7,51 & Univ. Trento \\
\hline Quickbird & 17 Jul 2006 & $\begin{array}{c}\text { PAN } \\
\text { MS4 }\end{array}$ & $\begin{array}{c}0.6 \\
2.4\end{array}$ & 15,08 & 9,20 & Univ. Trento \\
\hline SPOT5-VEG & 31 Aug 2006 & MS4 & 10.0 & 36,28 & 36,13 & Univ. Trento \\
\hline SPOT5-HRG & 31 Aug 2006 & PAN & 2.5 & 36,22 & 36,09 & Univ. Trento \\
\hline WV2 & 22 Aug 2010 & PAN & 0.51 & 17,64 & 17,64 & FBK \\
\hline WV2 & 22 Aug 2010 & $\begin{array}{c}\text { PAN } \\
\text { MS8 }\end{array}$ & $\begin{array}{c}0.51 \\
2.0\end{array}$ & 17,64 & 17,64 & FBK \\
\hline GE1 & 28 Sep 2011 & $\begin{array}{c}\text { PAN } \\
\text { MS4 }\end{array}$ & $\begin{array}{c}0.50 \\
2.0\end{array}$ & 10,00 & 10,00 & FBK \\
\hline GE1 & 28 Sep 2011 & $\begin{array}{c}\text { PAN } \\
\text { MS4 }\end{array}$ & $\begin{array}{c}0.50 \\
2.0\end{array}$ & 10,00 & 10,00 & FBK \\
\hline
\end{tabular}

\section{PROCESSING OF VHR SATELLITE IMAGES}

The WV2 image pair was acquired 22 August 2010 at circa 10:40 am (GMT). The first image was recorded in forward scanning mode with an average in-track viewing angle of $15.9^{\circ}$, while the second one was acquired in reverse scanning mode with an average in-track viewing angle of $-14.0^{\circ}$. Cloud cover in both images is $10 \%$ or less. The forward-looking image was delivered in 4 tiles, while the backward-looking image was delivered in 5 tiles. A scale and radiometric example of changes between the two images is given in Fig. 2 (top).

The GE1 stereo pair was acquired 28 September 2011 at circa 10:20 am (GMT). Both images were recorded in reverse 
scanning position, with a nominal in-track viewing angle of about $15^{\circ}$ in forward direction and $-20^{\circ}$ in backward direction (Fig. 2, bottom). Cloud cover in both images is $4 \%$ or less. Some small errors occurred in the images (saturation, spilling), due to the presence of reflective surfaces, in combination with the incidence angles. Fig. 3 and Fig. 4 show some examples.
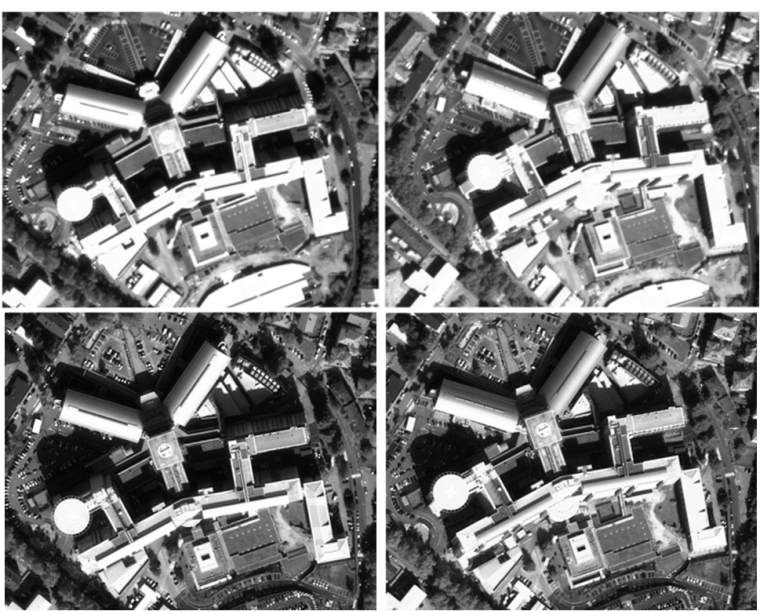

Figure 2. Zoom in the stereo satellite images on the same building block. [Top] WV2 forward-looking (left) and backward-viewing (right) images. [Bottom] GE1 forward (left) and backward (right) images.
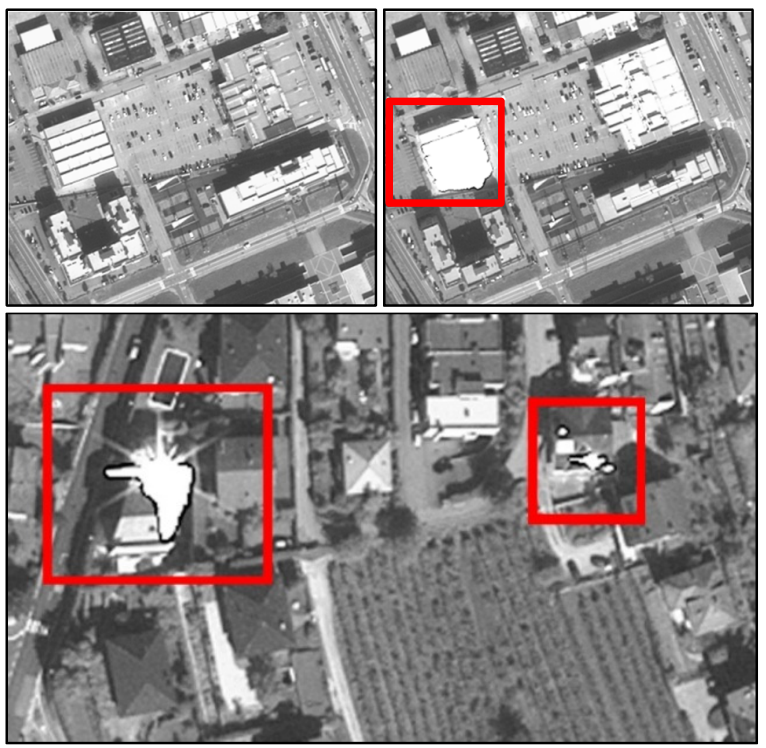

Figure 3. Examples of artefacts found in GE1 images.

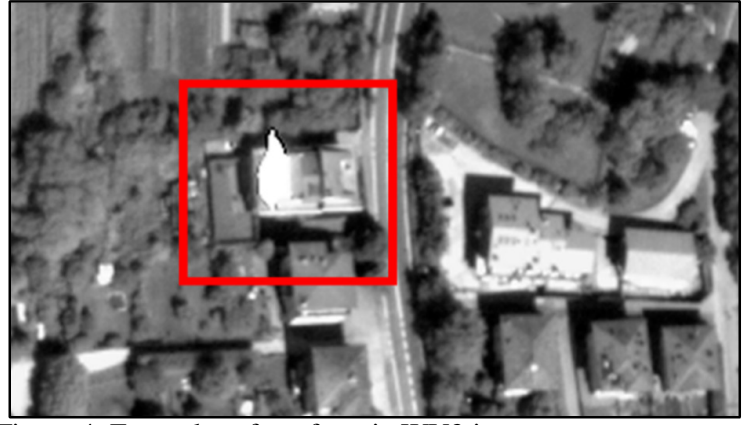

Figure 4. Examples of artefacts in WV2 images.

\subsection{Orientation and DSM generation}

For the geometric processing of the WorldView-2 and GeoEye1 stereo-scenes, the commercial software SAT-PP (SATellite image Precision Processing) by 4DiXplorer AG (http://www.4dixplorer.com) was used. Information about the software functionalities and the approaches for image orientation and DSM generation is given in Poli et al. (2009). The images were oriented based on the RPC-based model, by estimating the parameters modelling an affine transformation to remove systematic errors in the given RPCs. The estimation is run simultaneously for the two stereo images of each pair in a block adjustment. For this operation, a selection of available ground points visible in both images was used. A sub-pixel accuracy was reached in the orientation both for WV2 and GE1 stereo-pairs.

The DSMs were generated with a multi-image least-square matching, as described in Poli et al. (2009), using a grid space equal to 2 times the GSD, which leads to $1 \mathrm{~m}$ geometric resolution surface models. Few seed points were manually measured in the stereo images in correspondence of height discontinuities to approximate the surface. The DSMs were neither manually edited nor filtered after their generation. Fig. 5 shows the DSM obtained from GE1in its full extent and two zooms in the city centre and in hilly rural area.

For 3D realistic visualization, the GE1 panchromatic and multispectral channels of the forward image were pansharpened and orthorectified on the GE1 DSM. The result is shown in Fig. 6
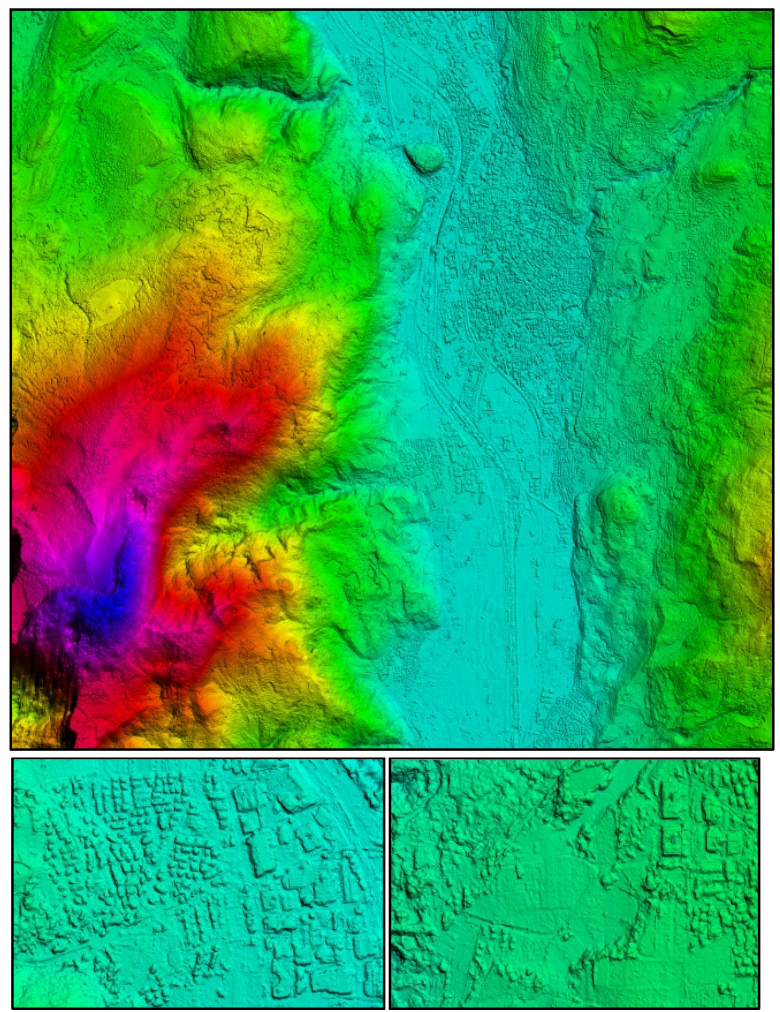

Figure 5. DSM from the GE1 stereo-pair. [Top] visualization in colour-shaded mode in SAT-PP software. [Bottom] zoom over Trento (left) and a steep rural area (right). 


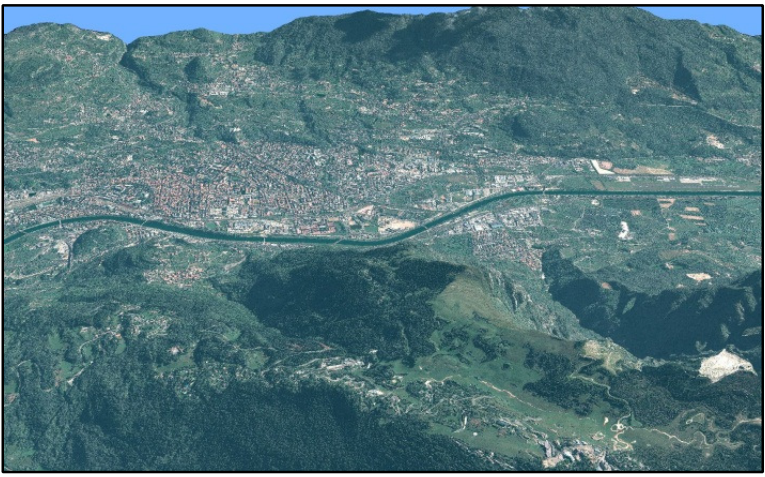

Figure 6. 3D visualization in ERDAS VirtualGIS of the textured model of Trento produced using GE1 stereo images.

\subsection{DSM quality analysis}

In general, the DSMs were successfully generated from WV2 and GE1 stereo images. In the mountain area on the west-south side of Trento, characterised by steep mountains and large height difference, the shape of valleys, mountain sides and ridges and even roads is well modelled. In urban areas, building agglomerations, blocks with different heights, the road network, some infrastructures (i.e. bridges), and rivers are well outlined. In rural areas, adjacent fields, vegetation, and buildings are recognised on flat or hilly terrain (Fig. 5).

For quality evaluation, we compared the WV2 and GE1 DSMs with the LiDAR one in three areas, and computed the statistics of the height differences, in terms of minimum and maximum values, mean value, standard deviation and root mean square error (RMSE). Table 2 shows the test area location, the reference LiDAR DSM, the GE1 and WV2 DSMs and the error maps, together with the frequency histograms and statistics.

In the error maps the height differences in the range [-1 $\mathrm{m}, 1 \mathrm{~m}]$ were plotted in white, as they are within the intrinsic precision of the sensors, while large positive and negative errors were highlighted in red (LiDAR above the DSM) and blue (DSM above LiDAR), respectively. From the analysis of the DSMs behaviour in the historic centre of Trento, characterised by small adjacent units and narrow streets, the height of the roofs is estimated quite well. Errors are encountered between buildings, as narrow streets are not visible in the stereo-pairs due to shadows or occlusions. In these cases the two DSMs are above the LiDAR one, as they do not model the street level. The two large red spots (highlighted in red and blue in the LiDAR DSM) occur on churches, both in GE1 and WV2 DSMs. The matching failure is likely due to the homogeneous material used for the roof cover, the shadowing, and, eventually, the structure geometry. The manual measurements of seed points on the two buildings would certainly help the matching procedure. The values in the statistics confirm the analysis. The height profiles indicates that both DSMs follow well the LiDAR profile both on buildings and in open areas (i.e. square), being the GE1 DSM slightly more accurate than WV2 one. The matching error in correspondence of the church roof cover is evident in the profile, too. In the train station test area, again, there is a general agreement between the two DSMs and the LiDAR one. Some differences are due to the presence of trees (blue circle, LiDAR is below the DSMs) and to a change in the area (red circle, LiDAR is above the DSMs), that is, a building was demolished between LiDAR and WV2/GE1 acquisitions. Regarding the third test area (Trento Fersina), significant height differences occur in correspondence of trees (blue areas) and between tall buildings. The height profiles in one problematic area confirm that the tall buildings are identified in the DSMs as height peaks, but they are not completed separated, as the area between them is occluded in the images or in shadow (Fig. 7). The error frequency histograms of GE1 and WV2 DSM follow a normal distribution, with small lateral peaks due to the mentioned errors. The quality of GE1 DSM seems slightly better than the WV2 one, as its histograms have smaller width and higher central peaks.
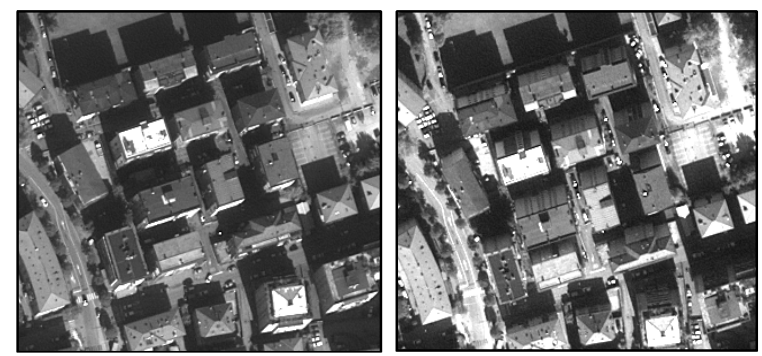

Figure 7. Example of occlusion between tall buildings in GE1 stereo-pair: forward view [left] and backward view [right].

\section{CONCLUSIONS AND OUTLOOK}

This paper presented the testfield set up in Trento by the 3D Optical Metrology (3DOM) Unit of Bruno Kessler Foundation (FBK) of Trento, Italy, with the aim to investigate optical imagery, perform automatic digital surface modelling, 2D/3D feature extraction and data fusion using state-of-the-art spaceborne, aerial and terrestrial data. With regard to spaceborne optical imagery, the testfield includes very high resolution (VHR) stereo imagery acquired by WoldView-2 and GeoEye-1 sensors. The images were processed with SAT-PP commercial software and two DSMs were extracted. The quality of the DSMs was assessed using a LiDAR DSM as reference. In general, the DSMs model well the surface in flat, hilly and mountainous areas, where cliffs, ridges and roads are well defined. In urban areas, building blocks are recognised in the historic city centre, characterised by small units closed to each other, and individual buildings are visible in residential and industrial areas. With respect to LiDAR, there is a general agreement. Significant differences (larger than $1.0 \mathrm{~m}$ ) between LiDAR and optical DSMs are encountered in case of (i) occlusions, mainly due to tall buildings or narrow streets, (ii) homogenous texture (shadows, special roof covers), (iii) temporal changes between LiDAR and WV2/GE1 images, (iv) temporary objects like construction sites, and (v) presence of vegetation (trees, forests), where optical sensors and LiDAR respond differently. The statistics on three test areas shows mean values close to zero and standard deviation in the range of $6-8 \mathrm{~m}$ due to the above mentioned problems. From a first analysis, the performance of GE1 and WV2 sensors is similar, but deeper investigations will be conducted.

The work on the VHR spaceborne resolution imagery in the Trento testfield is planned to be further extended by carrying out more radiometric investigations, DSM generation using other matching approaches and DSM analyses using a reference DSM from other optical sensors and excluding areas with temporal changes.

The other aerial images of the testfield Trento are currently being processed in order to evaluate automated aerial triangulation methods, image-matching algorithms, man-made feature extraction procedures (Nex and Remondino, 2012) and finally to estimate the PV potential on building roofs (Agugiaro et al., 2012). 
International Archives of the Photogrammetry, Remote Sensing and Spatial Information Sciences, Volume XXXIX-B1, 2012 XXII ISPRS Congress, 25 August - 01 September 2012, Melbourne, Australia

Table 2. Quality evaluation of WV2 and GE1 DSMs with respect to the LiDAR DSM: orthophoto with test areas contour (light blue) and profile transect (yellow), LiDAR DSM, GE1 DSM, WV2 DSM, error planimetric distribution, error histograms (GE1 in blue, WV2 in green), statistics (minimum, maximum, mean $\mu$, standard deviation $\sigma$, RMSE) and height profiles. Measures are in metres.

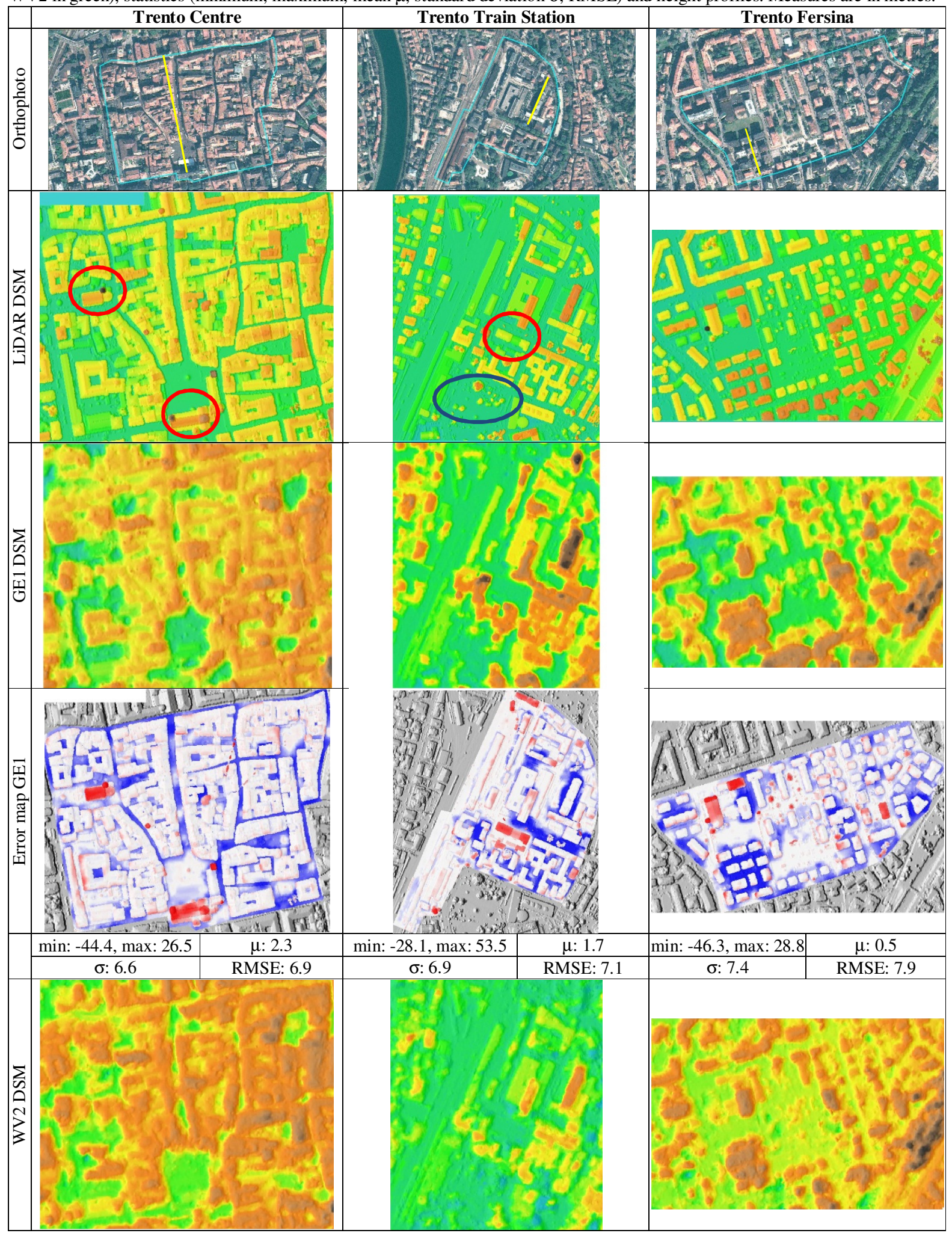




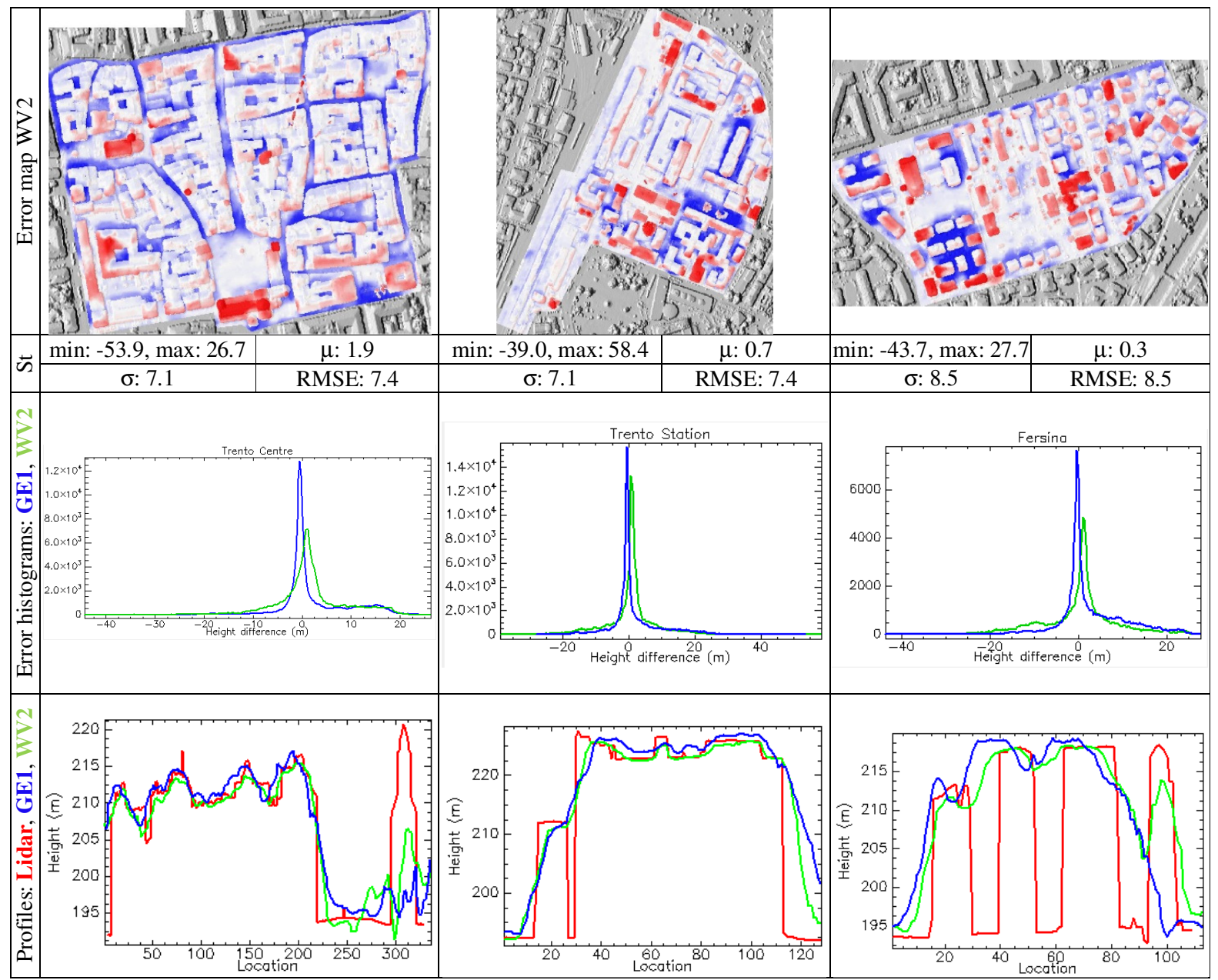

\section{REFERENCES}

Agugiaro, G., Nex, F., Remondino, F., Droghetti, S., De Filippi, R., Furlanello, C., 2012. Solar radiation estimation on building roofs and web-based solar cadastre. ISPRS Annals of Photogrammetry, Remote Sensing and Spatial Information Sciences, XXII ISPRS Congress, Melbourne, Australia.

Arefi, H., Reinartz, P., 2011. Building reconstruction from Worldview DEM using image information. Proc. Int. Conference on Sensors \& Models in Remote Sensing \& Photogrammetry, SMPR 2011, Tehran, Iran, (on CD-ROM).

Capaldo, P., Crespi, M., Fratarcangeli, F., Nascetti, A., Pieralice, F., 2012. DSM generation from high resolution satellites imagery: applications with WorldView-1 and GeoEye-1. Italian Journal of Remote Sensing, Vol. 44, pp. 41-53.

d'Angelo, P., Reinartz, P., 2011. Semiglobal matching results on the ISPRS stereo matching benchmark. Int. Archives of Photogrammetry and Remote Sensing and Spatial Information Sciences, Vol.38(4/W19), (on CD-ROM).

Krauss, T., Lehner, M., Reinartz, P., 2008. Generation of coarse 3D models of urban areas from high resolution stereo satellite images. Int. Archives of Photogrammetry and Remote Sensing and Spatial Information Sciences, Vol. 37(B1), pp. 10911098. ISSN 1682-1750.

Nex, F., Remondino, F., 2012. Automatic roof outlines reconstruction from photogrammetric DSM. ISPRS Annals of Photogrammetry, Remote Sensing and Spatial Information Sciences, XXII ISPRS Congress, Melbourne, Australia.
Poli, D., Wolff, K., Gruen, A., 2009. Evaluation of Worldview1 stereo scenes and related 3D products. ISPRS Hannover Workshop 2007, Int. Archives of the Photogrammetry, Remote Sensing and Spatial Information Sciences, Vol. 38(14-7/W5).

Poli, D., Angiuli, E., Remondino, F., 2010. Radiometric and geometric analysis of Worldview-2 stereo scenes. Int. Archives of Photogrammetry and Remote Sensing and Spatial Information Sciences, Vol. 38(1), Calgary, Canada, (on CDROM).

Poli, D., Caravaggi, I., 2012. 3D information extraction from stereo VHR imagery on large urban areas: lessons learned. Natural Hazards, (in press)

Poli, D., Toutin, Th., 2012. Developments in geometric modelling for HR satellite push-broom sensors. The Photogrammetric Record, Vol. 27(137), pp. 58-73.

\section{ACKNOWLEDGEMENTS}

This work was partly supported by the $3 \mathrm{M}$ project (co-founded Marie-Curie Actions FP7 - PCOFOUND - GA-2008-226070, acronym "Trentino Project", Bando "Incoming Team 2009"). The authors would also like to thank the Autonomous Province of Trento (PAT) and the Municipality of Trento, for providing spatial data for the testfield, as well as Prof. Bruzzone and his research team at RSLab (University of Trento, Italy, web: http://rslab.disi.unitn.it) for providing information about the SPOT and the Quickbird imagery. 